\title{
Severidad de la inflamación en los reportes de Papanicolaou según agente etiológico encontrado en un hospital público del norte de Perú
}

\author{
Susy Bazán-Ruiz1,a, Danai Valladares-Garrido2,a, Yamalí Benites-Cóndor3,a, Guido \\ Bendezú-Quispe 4,b, Muchín Ruiz-Leud5,c, Christian R. Mejia.6,a \\ ${ }^{1}$ Universidad César Vallejo - filial Piura. ${ }^{2}$ Unidad de apoyo a la docencia e investigación (UADI), Hospital de Apoyo II-2, \\ Sullana, Piura. ${ }^{3}$ Universidad Privada Antenor Orrego, Piura. ${ }^{4}$ Universidad Nacional San Luis Gonzaga, Ica. ${ }^{5} \mathrm{Hospital}$ de \\ la Amistad Perú-Corea II-2 Santa Rosa, Piura. ${ }^{6}$ Escuela de Medicina Humana, Universidad Continental. Huancayo, Perú.
}

$\mathrm{a}_{\text {Médico Cirujano. }}{ }^{\mathrm{b}}$ Estudiante de Medicina. ${ }^{\mathrm{C}}$ Médico Anátomo Patóloga.

\section{RESUMEN}

Antecedentes. Las infecciones de transmisión sexual son un problema de salud con implicancias diversas, los factores asociados varían desde el inicio de las relaciones sexuales, número de parejas, uso de métodos anticonceptivos, entre otros. Mediante la citología se puede evaluar los cambios inflamatorios y la presencia de agentes patógenos; la relación entre los factores antes mencionados es conocida pero poco estudiada aún. Objetivo: Determinar la asociación entre el grado de severidad del reporte y el agente etiológico encontrado en la citología cérvico-vaginal. Métodos: Estudio transversal analítico, de los reportes de Papanicolaous del consultorio de ginecología y obstetricia del Hospital de Apoyo-Sullana II-2, Piura-Perú, 2007-2011. Se realizó un muestreo por conveniencia tipo censal $(n=1535)$. Obteniendo datos del tipo de agente según el grado de inflamación, se realizó análisis descriptivos y analíticos, usando las pruebas chi2 y U de Mann Whitney. Resultados: La mediana de edades de las pacientes fue 36 años. Se encontró agentes patógenos en el 34\% de muestras; las más frecuentes fueron la Cándida (64\%) y la Gardnerella $(28 \%)$. Se encontró una fuerte asociación entre la severidad de la inflamación y la presencia de GardnereIla $(p<0,001)$, Trichomonas $(p<0,001)$, la co-infección de dos gérmenes $(p<0,001)$ y por cada año extra de edad de la paciente $(p<0,001)$. Conclusiones: La severidad del reporte de Papanicolau está asociada al tipo de germen, la co-infección y la edad de la paciente. Esto debe ser tomado en cuenta para el manejo terapéutico en poblaciones similares.

\section{PALABRAS CLAVE: Papanicolaou, citología cervical inflamatoria, Cándida, Trichomonas, Gardnerella}

\section{SUMMARY}

Background: Sexually transmitted infections are a health problem with many implications, associated factors, ranging from the beginning of intercourse, number of partners, contraceptive methods use, among others. By cytology can assess inflammatory changes and the presence of pathogens. The relationship between the above factors is known but there are not enough studies. Objective: To determine the association between the severity of the report and the etiologic agent found in the cervical-vaginal cytology. Methods: Cross-sectional study of Papanicolaous reports from 2007-2011 of obstetrics and gynecology's area at "Hospital de Apoyo II-2, Sullana", Piura, Peru. A convenience sampling census type ( $=1535)$ was 
performed to obtain type of agent's data according to the degree of inflammation; also descriptive and analytical analysis was performed using the tests chi-square and Mann Whitney. Results: The median age of patients was 36 years. Pathogens was found in $34 \%$; the most common were Candida (64\%) and Gardnerella (28\%). A strong association between the severity of inflammation and the presence of Gardnerella $(p<0.001)$ was found; Trichomonas $(p<0.001)$, the co-infection of two germs $(p<0.001)$ and for each additional year of age of the patient $(p<0.001)$. Conclusions: The severity of the report Papanicolaous is associated with the type of germ, co-infection and the age of the patient. This should be taken into account for the therapeutic management in similar populations.

\section{KEY WORDS: Papanicolaou, inflammatory cervical cytology, Candida, Trichomonas, Gardnerella}

\section{INTRODUCCIÓN}

Las infecciones de transmisión sexual (ITS) son un problema de salud pública mundial, la Organización Mundial de la Salud (OMS) refirió para el año 2008 un estimado de 498,9 millones de nuevos casos de ITS por Clamydiatrachomatis, Neisseria gonorrhoeae y Trichomonas vaginalis, lo que representa una estimación $11 \%$ más alta que la señalada para el año para 2005. Se conocen más de 30 agentes patógenos transmisibles, incluyendo bacterias, virus y parásitos; esto no solo genera la enfermedad, también discapacidad a largo plazo y hasta la muerte (1). Los factores de riesgo para adquirirlas son el tener actividad sexual sin protección, tener más de una pareja sexual o una considerada de alto riesgo sexual, entre las más destacadas $(2,3)$.

La citología cérvico-vaginal posee una sensibilidad alrededor del $50 \%$ y una especificidad alta para detectar lesiones de alto grado -e incluso el cáncer- $(4,5)$, pero también nos proporciona una información valiosa sobre el estado hormonal y la presencia de cambios inflamatorios, además de la presencia de agentes causantes de enfermedades de transmisión sexual.

Es conocida la asociación entre citología cérvico-vaginal inflamatoria con la lesión intra-epitelial cervical y el tipo de agente etiológico; la mayoría de las investigaciones en nuestro medio hacen referencia a los grados de severidad inflamatoria y agentes etiológicos predominantes $(6,7,8)$. Al no existir muchos reportes del grado de severidad de la infección según el tipo de agente, nos planteamos el objetivo de determinar la asociación entre el grado de severidad del reporte y el agente etiológico encontrado en la citología cérvico-vaginal.

\section{MATERIAL Y MÉTODOS}

Diseño y población: Estudio transversal analítico de datos secundarios, realizado en el Hospital de Apoyo II de la provincia de Sullana-Piura, Perú. Establecimiento referencial de las regiones Piura,
Tumbes y ámbito binacional peruano-ecuatoriano de la cuenca Catamayo - Chira. Además de ello, al ser un hospital público del Ministerio de Salud (MINSA), atiende personas de distintos sectores socio-económicos, siendo similar a otros hospitales de las regiones aledañas. La población considerada para el estudio estuvo conformada por los reportes de Papanicolaous (PAPs) de los años 2007 al 2011, procedentes del consultorio de ginecología y obstetricia. Se excluyó del estudio 47 reportes, por encontrarse registros incompletos o con información no clara (tasa de pérdida: $2 \%$ ). Se realizó un muestreo por conveniencia de tipo censal, ya que se usaron la totalidad de los reportes realizados en el período de estudio.

Variables: La variable dependiente principal fue la severidad del reporte de PAP, que fue consignada por los anátomo-patólogos del hospital, que tuvieron como guía para dicha detección el considerar el grado de infiltrado de polimorfonucleares en la observación al microscopio. Se homogenizó el hallazgo de los distintos patógenos, para la determinación de la infección por Cándida albicans, los expertos debían observar en el microscopio numerosas pseudo hifas y formas de levaduras extracelulares. Para la identificación de Trichomonas se encontró un frotis en vidrio esmerilado de color verde grisáceo y se identificó su característico núcleo oval y excéntrico, rechazado a la periferia. Se consideró infección por Gardnerella al frotis de color basófilo (azul-grisáceo) con bacilos cortos intracelulares adheridas al citoplasma de las células respetando al núcleo y formando así las características "células clave" "clue cells" o "células guía". Por último, el diagnóstico de infección por Leptothrix fue ante la identificación de bacilos largos de color gris extracelulares. También se consideró la edad de la paciente consignada en el reporte (tomada como variable cuantitativa), año de toma de muestra (variable cuantitativa) y agente etiológico encontrado mediante microscopia.

Procedimientos: Se presentó una solicitud a la jefatura del área de Patología del Hospital de Apoyo II de Sullana, con la finalidad de tener acceso al libro 
de registro citopatológico de los PAP's. Obtenida su aprobación se accedió a las cifras de la cantidad de reportes durante el periodo de estudio, luego se procedió a una revisión de los reportes de PAP's y la recolección de las variables. Los datos obtenidos fueron registrados en una base de datos diseñada en el programa Microsoft Excel 2007, que posteriormente fue exportada al paquete estadístico Stata versión 11,1 (Stata Corporation, College Station, TX). Análisis de datos: Para el análisis de datos univariados de las variables categóricas se usó las frecuencias y porcentajes, para las variables cuantitativas se analizó la normalidad mediante la prueba estadística Shapiro Wilk, reportando la mediana y rangos en todos los casos, por su distribución no normal. En el análisis bivariado se empleó la prueba del chi2 para el cruce de dos variables categóricas y la U de Mann Withney para las variables categóricas versus las cuantitativas. Para el análisis bivariado se encontraron las razones de prevalencia (RP) y sus intervalos de confianza al 95\% (IC95\%). Se usó los modelos lineales generalizados (GLM) con la familia binomial, la función de enlace log y modelos robustos, para ajuste por muestras grandes. Se consideró un valor $p<0,05$ como estadísticamente significativo.

Ética. Se contó con el permiso de la oficina de investigación del Hospital. En el análisis de datos secundarios no se usaron los datos personales de las pacientes, manteniendo la confidencialidad de los datos. Se respetó las normas éticas vigentes basadas en el reporte Beltmont.

\section{RESULTADOS}

En el período de estudio se tuvieron 2325 reportes de PAP's, la mediana de las edades de las pacientes fue 36 años (rango: 14-84 años). La frecuencia de agentes patógenos fue del $34,1 \%$. Siendo los gérmenes más comunes la Cándida (64\%) y la Gardnerella (28\%). Los otros agentes encontrados se reportan en la Tabla I.

Respecto al grado de inflamación reportado, se encontró severidad en casi la mitad de las muestras $(47 \%)$, seguido de grado moderado y leve, con el $36 \%$ y $17 \%$, respectivamente. En las muestras que indicaron presencia de Cándida, el grado de inflamación moderado y severo tuvo valores similares (42\% y $40 \%$, respectivamente). En el caso de Gardnerella, el grado de inflamación severa en el reporte se presentó en el $55 \%$, mientras que en los reportes con presencia de Trichomonas la inflamación severa estuvo presente en el $88 \%$ de los casos (Figura 1).

\section{Tabla I \\ HALLAZGO DE PATÓGENO ENCONTRADO EN LOS REPORTES DE PAPANICOLAOU DEL HOSPITAL DE PIURA-SULLANA, 2007-2011}

\begin{tabular}{lcc}
\hline Hallazgo patológico & $\mathrm{n}$ & $(\%)$ \\
\hline Cándida & 503 & 63,6 \\
Cándida-Trichomona & 2 & 0,3 \\
Cándida-Gardnerella & 9 & 1,1 \\
Cándida-Leptrotrix & 3 & 0,4 \\
Gardnerella & 227 & 28,4 \\
Leptrotrix & 13 & 1,8 \\
Trichomona & 31 & 3,9 \\
Trichomona-Gardnerella & 5 & 0,6 \\
\hline Total & 793 & 100 \\
\hline
\end{tabular}

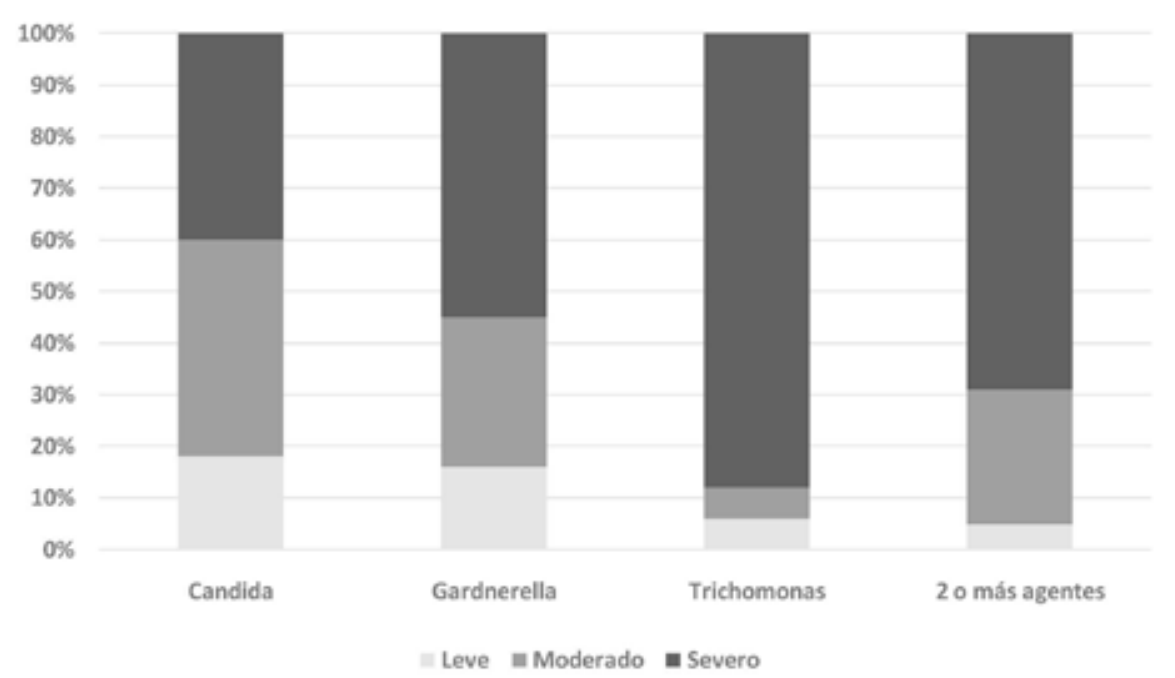

Figura 1. Grado de inflamación y agente encontrado en reportes de citología cérvico-vaginal. 
Al realizar el análisis bivariado de los tipos de patógenos según el grado de severidad se encontró una fuerte asociación entre la severidad de la inflamación y la presencia de Gardnerella $(p<0,001$; PRc: 1,6; IC95\%: 1,36-1,77); Trichomonas ( $p<0,001$; PRc: 2,4; IC95\%: 2,07-2,77), la co-infección de dos gérmenes ( $p<0,001$; PRc: 1,9; IC95\%: 1,36-2,53) y según la edad de manera cuantitativa $(\mathrm{p}<0,001$; PRc: 0,99; IC95\%: 0,98-0,99). No hubo asociación de la severidad con la infección por Cándida $(p=0,105)$ (Tabla II).

\section{DISCUSIÓN}

Se encontró asociación entre el grado de severidad y las infecciones vaginales más comunes según el PAP, esto es importante ya que al identificar los agentes responsables de un mayor grado de inflamación se podría, mediante la clínica, elegir el manejo terapéutico más idóneo; siendo esto importante en el primer nivel de atención, donde se debe tener el diagnóstico mediante una prueba rápida, económica y fiable, ya que por las características sociodemográficas, la opción de derivar a un centro especializado es casi nula.

Se encontró diferencia entre las prevalencias de los agentes etiológicos más comunes, la vaginosis bacteriana tiene una etiología predominantemente conformada por Gardnerella, encontrándose una prevalencia similar de casos de este agente en estudios realizados en la ciudad de Bolívar y otros en Estados Unidos, pero esta prevalencia es mayor que los resultados encontrados en Colombia y Perú, donde se reportaron frecuencias promedio de $13 \%(9,10)$.

En lo que respecta a la infección por Cándida albicans, los resultados de este estudio son ampliamente superiores que los encontrados en los estudios antes mencionados, que reportaron entre $5-39 \%(11,12,13)$. Sin embargo, cabe resaltar que existen variaciones en las prevalencias de los agentes encontrados por el PAP en comparación con estudios realizados en la ciudad de México, donde la candidiasis vaginal varía entre $10-25 \%$ (14,15-17).

En nuestro estudio se encontraron agentes patógenos en $32 \%$ de las muestras, lo cual es similar al encontrado en un estudio en Chile donde se reportó un $32 \%$ (18) y en Cuba un $20 \%$ (19), en los cuales se analizó una amplia población (mujeres embarazadas, en edad fértil y postmenopáusicas). Nuestros resultados sobre la prevalencia de agentes patógenos difieren con el encontrado en una población colombiana donde la afección principal fue la vaginitis con un $61 \%$ seguida de vaginosis con un $30 \%$ de casos en una población urbana (20).

\section{Tabla II
ANÁLISIS BIVARIADO DE LA RELACIÓN ENTRE HALLAZGO PATÓGENO Y SEVERIDAD DE LA INFLAMACIÓN}

\begin{tabular}{|c|c|c|c|c|}
\hline \multirow[t]{2}{*}{ Germen } & \multicolumn{2}{|c|}{$\begin{array}{c}\text { Severidad } \\
\mathrm{n}(\%)\end{array}$} & \multirow[t]{2}{*}{ Valor $p$} & \multirow[t]{2}{*}{ RP (IC95\%) } \\
\hline & Sí & No & & \\
\hline \multicolumn{5}{|l|}{ Cándida: } \\
\hline $\mathrm{Si}$ & $202(23,4)$ & $301(20,6)$ & 0,105 & $1,1(0,98-1,25)$ \\
\hline No & $661(76,6)$ & $1161(79,4)$ & & \\
\hline \multicolumn{5}{|l|}{ Gardnerella: } \\
\hline $\mathrm{Si}$ & $124(14,4)$ & $103(7,1)$ & $<0,001$ & $1,6(1,36-1,77)$ \\
\hline No & $739(85,6)$ & $1359(92,9)$ & & \\
\hline \multicolumn{5}{|l|}{ Trichomona: } \\
\hline $\mathrm{Si}$ & $27(3,1)$ & $4(0,3)$ & $<0,001$ & $2,4(2,07-2,77)$ \\
\hline No & $836(96,9)$ & $1458(99,7)$ & & \\
\hline \multicolumn{5}{|l|}{ Co-infección: } \\
\hline $\mathrm{Si}$ & $13(1,5)$ & $6(0,4)$ & $<0,001$ & $1,9(1,36-2,53)$ \\
\hline No & $850(98,5)$ & $1456(99,6)$ & & \\
\hline \multicolumn{5}{|l|}{ Edad (años) } \\
\hline Media $( \pm \mathrm{DE})$ & $35,5(0,4)$ & $38,6(0,4)$ & $<0,001$ & $0,99(0,98-0,99)$ \\
\hline
\end{tabular}

Valor p y razón de prevalencias (RP)/ intervalo de confianza al 95\% (IC95\%) obtenidas con modelos lineales generalizados, con familia binomial, función de enlace log y modelos robustos. 
Respecto a la infección por trichomoniasis es importante resaltar la presencia de inflamación severa en la gran mayoría de ellas, ya que se conoce que la trichomoniasis, como mínimo, duplica el riesgo de contagio con el VIH (21) y del VPH (22), ya que el sistema inmune, al ser atacado, activa los receptores tipo Toll, lo que induce la liberación de citocinas pro-inflamatorias y quimiocinas. Estas a su vez atraen y activan las células presentadoras de antígeno y los linfocitos TCD4+, siendo estos últimos infectados por el VIH-1 (21).

En lo que respecta a Gardnerella vaginalis, se reporta una prevalencia de entre 15 y $30 \%$ en mujeres en edad reproductiva (23).

Se encontró una fuerte asociación entre severidad de la enfermedad y los agentes etiológicos, no se encontró referencias concluyentes de esta asociación, pero existen algunos reportes de frecuencias, como el realizado por López y cols en el 2001 (24), que encontraron que el 78,8\% tenían infección cérvico-vaginal, de los cuales el 33,9\% de las infecciones era causada por dos o más microorganismos. Se evidenció que las mujeres con infección genital tienen etiología mixta $(94,8 \%)$ y el microorganismo más común fue Trichomona vaginalis, en combinación con la flora bacteriana.

En un estudio realizado en República Dominicana hubo predominancia de inflamación moderada a severa en el frotis de PAP, causada en su mayoría por $\mathrm{H}$. vaginalis, Cándida albicans y Trichomonas vaginalis en un tercer lugar (25).

Un estudio en Venezuela (26), mostró que la mayoría de los resultados de tipo inflamatorio fueron leves y el agente predominante fue Gardnerella, la presencia predominante de este agente en el tipo inflamatorio leve es un hallazgo contrario al encontrado por el presente trabajo. Así mismo, en un estudio realizado en una clínica rural de Santo Domingo (25), se encontró que el $26,2 \%$ tenía un frotis de PAP correspondiente a inflamación leve, 37,7\% inflamación moderada y $21,2 \%$ inflamación acentuada; la flora vaginal predominante fue mixta con un $36,1 \%$ de Haemophilus vaginalis, $22,9 \%$ de Cándida albicans y $21,3 \%$ de Trichomonas. En el caso de Trichomonas, el estudio realizado por Bertolino y cols (27), indica que Trichomonas es más común en pacientes cuyo Papanicolaou muestra presencia y aumento de inflamación, lo que guarda correspondencia con lo encontrado en el presente estudio.

Como limitación del estudio se debe señalar que el PAP es un examen operador dependiente, considerado como de baja sensibilidad, siendo en nuestro medio el único disponible, ya que no se cuenta con el método más apropiado, como cultivo en los caldos de Roiron y de Diamond (sensibilidad de $98 \%$ y especificidad de $100 \%$ ), InPouch ${ }^{\circledR}$ TV entre otros. A pesar de ello los resultados obtenidos son altos en relación a los antecedentes ya mencionados.

\section{CONCLUSIÓN}

La severidad del reporte de Papanicolaou está asociada al tipo de germen, la co-infección y la edad de la paciente. Esto debe ser tomado en cuenta para el manejo terapéutico en poblaciones similares.

AGRADECIMIENTOS: Los autores desean agradecer a la Sociedad Científica Médico-Estudiantil Peruana (SOClMEP), institución donde se generó la idea y los contactos para ésta investigación. Ésta investigación ocupó el sexto puesto en el concurso de trabajos científicos del XXVIII Congreso Científico Internacional de la Federación Latinoamericana de Sociedades Científicas de Estudiantes de Medicina (FELSOCEM), Honduras 2013.

\section{REFERENCIAS}

1. World Health Organization, Dept. of Reproductive Health and Research. Global incidence and prevalence of selected curable sexually transmitted infections. WHO [Internet]. 2008. Disponible en: http://www.who. int/reproductivehealth/publications/rtis/stisestimates/ en/

2. Bradshaw CS, Morton AN, Garland SM, Morris MB, Moss LM, Fairley CK. Higher-Risk behavioral practices associated with bacterial vaginosis compared with vaginal candidiasis. Obstet Gynecol 2005;106(1):10514.

3. González-Pedraza A, Mota R, Ortiz A, Ponce RE. Factors of risk of bacterial vaginosis. Atención Primaria / Sociedad Española de Medicina de Familia y Comunitaria2004; 34(7):360-5.

4. Fahey MT, Irwig L, Macaskill P. Meta-analysis of pap test accuracy. Am J Epidemiol 1995;141(7):680-9.

5. Nanda K, McCrory DC, Myers ER, Bastian LA, Hasselblad V, Hickey JD, et al. Accuracy of the papanicolaou test in screening for and follow-up of cervical cytologic abnormalities: a systematic review. Ann Intern Med 2000;132:810-9.

6. Dimian C, Nayagam M, Bradbeer C. The association between sexually transmitted diseases and inflammatory cervical cytology. Genitourin Med 1992;68(5):3056.

7. Baka S, Tsirmpa I, Chasiakou A, Tsouma I, Politi $\mathrm{E}$, Gennimata V, et al. Inflammation on the cervical papanicolaou smear: evidence for infection in asymptomatic women? Infectious Diseases in Obstetrics and Gynecology [Internet]. 2013 [citado el 05 abril 2015]; 4 paginas. Disponible en: http://dx.doi. org/10.1155/2013/184302

8. Bertolino JG, Rangel JE, Blake RL Jr, Silverstein D, Ingram E. Inflammation on the cervical Papanicolaou smear: the predictive value for infection in asymptomatic women. Fam Med 1992;24(6):447-52.

9. Hernandez E. Prevalencia de vaginitis y vaginosis bacteriana en personal policial de la provincia de Ica, Perú. Rev Méd Panacea 2011;1(2):40-3.

10. Angel-Müller $E$, Rodríguez A, Núñez-Forero LM. Prevalencia y factores asociados a la infección por $\mathrm{C}$. trachomatis, N. gonorrheae, T. vaginalis, C. albicans, sífilis, VIH y vaginosis bacteriana en mujeres con 
síntomas de infección vaginal en tres sitios de atención de Bogotá, Colombia, 2010. Rev Colomb Obstet Ginecol 2012;63(1):14-24.

11. Navarro A. Frecuencia de infecciones cervicovaginales. San José de Bongo. Estado Bolívar [Tesis]. Ciudad Bolívar: Universidad de Oriente, Núcleo Bolívar, Escuela de Ciencias de la Salud. Escuela de Ciencias de la Salud; 2010. Disponible en: http://ri.bib.udo.edu. ve/bitstream/123456789/2210/1/45\%20Tesis.\%20 WP9\%20W322.pdf

12. Nyirjesy P. Vulvovaginal candidiasis and bacterial vaginosis. Infect Dis Clin N Am 2008;22:637-52.

13. Cardona-Arias Jaiberth Antonio, Herrera-Posada Daniela, Valencia-Arredondo Marleny. Prevalence of positive cytology results for bacterial vaginosis, candidiasis and trichomoniasis in a Social State Institution in Medellín, Colombia, 2010-2012. Rev Colomb Obstet Ginecol 2014; 65(3):206-214.

14. Gonzales-Pedraza A, Ortiz Z, Davila MR, Valencia $\mathrm{CM}$, et al. Infecciones cervicovaginales más frecuentes; prevalencia y factores de riesgo. Rev Cubana Obstet Ginecol 2007;33(2):1-12.

15. Bradshaw CS, Morton AN, Garland SM, et al. Higherrisk behavioral practices associated with bacterial vaginosis compared with vaginal candidiasis. Obstet Gynecol 2005;106(1):105-14.

16. Klufio CA, Amoe AB, Delamare O, Hombhanje M, Kariwiga G, Igo J. Prevalence of vaginal infections with bacterial vaginosis, Trichomonas vaginalis and Candida albicans among pregnant women at the Port Moresby General Hospital Antental Clinic. P N G Med J 1995;38(3):163-71.

17. González Pedraza A, Mota VR, Ortiz ZC, Ponce RR. Factores de riesgo asociados a vaginosis bacteriana. Aten Primaria 2004;34(7):360-5.

18. Lillo E, Lezama S, Medel J, Martínez A. Diagnóstico de vaginosis bacteriana en un consultorio de planificación familiar de la región metropolitana Chile. Rev Chil Infect 2010;27(3):199-203.
19. Gonzales A, Ortiz C, Dávila R, Valencia C. Infecciones cervicovaginales más frecuentes; prevalencia y factores de riesgo. Rev Cubana Obstet Ginecol 2007;33(2):1-12.

20. Angel Müller E, Gonzales M P, Nuñez L et al. Frecuencia de infecciones del tracto genital femenino en mujeres sintomáticas y uso de pruebas rápidas para su diagnóstico en dos poblaciones de Bogotá (Colombia) 2008. Estudio piloto. Rev Colomb Obstet Ginecol 2010;61:220-30.

21. Mavedzenge SN, Pol BVD, Cheng $H$, Montgomery $\mathrm{ET}$, Blanchard K, de Bruyn G, et al. Epidemiological synergy of Trichomonas vaginalis and HIV in Zimbabwean and South African women. Sex Transm Dis 2010;37(7):460-6.

22. Huppert JS. Trichomoniasis in teens: an update. Curr Opin Obstet Gynecol 2009;21(5):371-8.

23. Flores R, Martínez R, Martín J. Prevalencia de vaginosis bacteriana en una Clínica Universitaria. RevFacultad Salud PubNutr 2003;4(1):1. Disponible en: http://www.respyn.uanl.mx/iv/1/articulos/vaginosis. html

24. López M, Toro M, Guillén M. Citología de las infecciones cervicovaginales. Rev Facultad Farm 2001;42(1). Disponible en: http://www.saber.ula.ve/ bitstream/123456789/23781/1/articulo42-16.pdf

25. Infante Reyes A, Martínez A, Suárez Paredes A. Hallazgos citológicos en muestras de cuello uterino (papanicolau) en pacientes de la Clínica Periférica Pueblo Nuevo, Baní, Provincia Peravia, enero-noviembre del año. Rev MédicaDominicana 1996;57(1/3):24-6.

26. Tagliaferro Zulay A,Villegas $F$,Uribe $C$,Valderrama E. Frecuencia de hallazgos citológicos y factores de riesgo asociados a patologías cervico uterinas. San Miguel, Municipio Jiménez, Estado Lara, Mayo 2004. Boletín Médico de Postgrado 2004;20(2):93-8.

27. Bertolino JG, Rangel JE, Blake RL Jr, et al. Inflammation on the cervical Papanicolaou smear: the predictive value for infection in asymptomatic women. Fam Med 1992;24(6):447-52. 